\title{
Maxilla Voluminous Bone Sequesters: About 4 Clinical Cases Treated at CCTOS in CHU Cocody
}

\author{
Patrice Attogbain Kouamé1 ${ }^{*}$, Daniel Amantchi', Akpe Jonas Adou ${ }^{1}$, \\ Wendpouiré Patrice Laurent Guiguimdé2, Vazoumana Kouyaté ${ }^{1}$, Nguessan Koboh Atsé ${ }^{1}$ \\ ${ }^{1}$ Department of Surgery, Pathology and Therapeutics, Anesthesiology, Resuscitation, Radiology UFR of Odonto-Stomatology, \\ Houphouët-Boigny University of Abidjan, Abidjan, Ivory Coast \\ ${ }^{2}$ Training and Research Unit in Health Sciences (UFR/SDS), Joseph Ki Zerbo University, Ouagadougou, Burkina Faso \\ Email: *attogbain@yahoo.fr
}

How to cite this paper: Kouamé, P.A., Amantchi, D., Adou, A.J., Guiguimdé, W.P.L., Kouyaté, V. and Atsé, N.K. (2021) Maxilla Voluminous Bone Sequesters: About 4 Clinical Cases Treated at CCTOS in CHU Cocody. Open Journal of Stomatology, 11, 451459.

https://doi.org/10.4236/ojst.2021.1111040

Received: September 27, 2021

Accepted: November 20, 2021

Published: November 23, 2021

Copyright (c) 2021 by author(s) and Scientific Research Publishing Inc. This work is licensed under the Creative Commons Attribution International License (CC BY 4.0).

http://creativecommons.org/licenses/by/4.0/

\begin{abstract}
Introduction: Jaw osteitis is an inflammatory disease of bone tissue causing aseptic necrosis of bone. This study aims to clarify the etiologies of osteitis of the jawbones and to describe the clinical and radiographic aspects of extensive forms. Cases Presentation: Our study concerns the clinical and radiographic examination of 4 cases of voluminous bone sequesters. The causes of osteitis are mainly infectious and due to late consultations of our populations. The development of these maxillary osteitis leads to the formation of large bone sequesters. Conclusion: The evolution towards voluminous sequesters is a combination of dental infection, poor oral hygiene, late consultation and traditional treatment.
\end{abstract}

\section{Keywords}

Osteitis, Sequesters, Jawbones, Late Consultation

\section{Introduction}

Osteitis of the maxilla is an inflammatory disease of the bone tissue. It is frequent in developing countries. The osteitis is responsible for a significant mortality rate, but above all for major aesthetic, functional and psychological complications that degrade the quality of life of patients. In Côte d'Ivoire, a study has shown that the prevalence of osteitis is $66.7 \%$ and concerns mostly patients under 30 years old [1]. Several etiologies have been mentioned in the scientific literature. In our developing countries, those resulting from complications of alveolo-dental infections are essen- 
tially evoked in the context of late consultations, traditional therapies and inaccessibility to health care. Anything that favors the installation of septic necrosis of the bone with the formation of voluminous alveolo-dental sequesters. The aim of this work is to report the clinical and therapeutic aspects of 4 clinical cases of osteitis of the maxilla in the surgical department of the Centre de Consultation et de Traitements Odonto-Stomatologiques (CCTOS) of the CHU Cocody.

\section{Cases Presentation}

This work concerns 4 clinical cases of alveolar-dental bone sequesters treated in the surgical department of the Centre de Consultation et de Traitements Odonto-Stomatologiques (CCTOS) of the CHU Cocody. The sociodemographic, clinical, radiographic and therapeutic data of these different cases are presented below.

Clinical Case 1: The patient K.L., 10 years old, came in consultation for a left genital swelling of dental origin. He had been treated for over a year with tree bark decoctions without success. The interrogation revealed no pathological antecedents. The patient's general condition was good. On endobuccal examination, halitosis, gingivorrhages, absence of 64 and 65 and their exposed alveolar bones were noted. The cusps of 24 and 25 are present in the focus and there is mucosal dehiscence in 26 . The panoramic radiograph performed showed significant osteolysis extending from the 63 to the distal aspect of the 26. Biological examinations were performed: The blood count showed microcytic hypochromic anemia associated with leuko-lymphocytosis, negative retroviral serology, and normal blood glucose, prothrombin and activated partial thromboplastin levels.

Clinical Case 2: The patient K.F., 7 years old, younger sister of case 1, came to consult for a left mandibular pain. Patient had good general condition and do not have pathological antecedents. The pathology started 2 years ago with a dental caries on the 36th tooth. Untreated and in a context of self-medication and tradipratic (use of traditional medicinal plant decoctions), this caries was complicated by a cellulitis and then an osteitis.

The clinical examination revealed a genial swelling, a painless adenopathy under the left mandible and poor oral hygiene. In addition, a bone exposure, mobile sequester extended from 73 to 36 .

Clinical Case 3: A 7 years old patient came for a genial swelling that appeared more than 6 months ago following an untreated carious process on the $75 . \mathrm{Pa}$ tient had good general condition. Notions of recurrent abscesses treated by the use of decoction were evoked. On inspection, there is a low genial swelling with the presence of skin fistulas, a left sub-maxillary adenopathy and a normal mouth opening. Endobuccal examination revealed poor oral hygiene and a mobile bony sequestrum extending from 73 to 36 . Orthopantomography performed showed osteolysis from the germ of 33 to 36 .

Clinical Case 4: The fourth case is that of a 38-year-old woman who came to the clinic for odontalgia and for aesthetic and functional reasons. Patient had 
good general condition. The pathology started with a caries on a right mandibular molar. After several episodes of acute painful phases and remissions, multiple cutaneous-mucosal fistulas appeared, followed by spontaneous exteriorization of a bone sequestrum picked by the patient. This was the patient's very first consultation with a dental surgeon. On inspection, right facial asymmetry and the presence of unsightly and retractile fistula scars under the right and left chin and angulomandibular areas are noted. In addition, we note the presence of sub-angulomandibular adenopathies, a normal mouth opening amplitude with a gagging path. Endobucally, poor oral hygiene is very marked with the presence of tartar blocks covering the mandibular and maxillary teeth, strong halitosis, gingival inflammation, mucosal budding and mucosal ulcerations in places. The teeth appeared trapped in calculus with significant mobile mandibular bone sequestration from 48 to 36 . All alveolar bone and remaining mandibular teeth were mobile. The orthopantomograph analysis shows images of bone rarefactions known as wet sugar covering the entire mandible, from 48 to 37 with telescoping of the dental elements. The mandibular bone is seriously eroded with a very thinned cortical bone in some places and a proximity between the dental canal and the pathological image.

The diagnosis of alveolar-dental bone sequester in these 4 clinical cases was made and management required surgical treatment, in this case sequestrectomies under local anesthesia followed by careful alveolar curettage. Sequestrectomy also facilitated extraction of teeth contained in the sequesters. The patients were treated with amoxicillin, metronidazole, paracetamol and chlorhexidine mouthwash. The patients were reviewed one week later and the postoperative course was normal.

The 4 clinical cases in this study concern 2 females and 2 males, including 3 children ( 7 years, 7 years and 10 years) and 1 adult of 38 years (Table 1 ).

The history reveals common characteristics in all subjects. These are reported notions of late consultation, pain, mucocutaneous fistulization, traditional practice and self-medication (Table 2).

On inspection during the physical examination, 3 cases presented with facial asymmetry (swelling). Perimaxillary palpation of the incriminated areas was painful in one subject, as was a limited mouth opening, thus producing a trismus. All subjects had poor oral hygiene (tartar deposits, dental caries), halitosis and mobility of the alveolar-dental sequesters. As for the complementary examinations, orthopantomogram and hemogram were performed. The bone sequester was mobile and of large span.

Table 1. Distribution of subjects by gender, age and location of sequestration.

\begin{tabular}{ccccc}
\hline Cases & $\mathrm{n}^{\bullet} \mathbf{1}$ & $\mathrm{n}^{\bullet} \mathbf{2}$ & $\mathrm{n}^{\bullet} \mathbf{3}$ & $\mathrm{n}^{\bullet} \mathbf{4}$ \\
\hline Gender, & Male, & Female, & Male, & Female, \\
Age & 10 years & 7 years & 7 years & 38 years \\
Location & Maxillary & Mandibular & Mandibular & Mandibular \\
\hline
\end{tabular}


Table 2. Distribution according to characteristics at history, physical examination, radiographic and therapeutic.

\begin{tabular}{|c|c|c|c|c|c|}
\hline \multicolumn{2}{|c|}{ Variables } & \multirow{2}{*}{$\begin{array}{c}\mathrm{n}^{\circ} 1 \\
+\end{array}$} & \multirow{2}{*}{$\begin{array}{c}\mathbf{n}^{\circ} 2 \\
+\end{array}$} & \multirow{2}{*}{$\begin{array}{c}\mathrm{n}^{\bullet} 3 \\
+\end{array}$} & \multirow{2}{*}{$\begin{array}{c}\mathrm{n}^{\circ} 4 \\
+\end{array}$} \\
\hline \multirow{6}{*}{ Characteristics of the history } & Pain & & & & \\
\hline & Swelling & + & - & + & + \\
\hline & Fistulization & + & + & + & + \\
\hline & Self medication & + & + & + & + \\
\hline & Tradipractic & + & + & + & + \\
\hline & Later consultation & + & + & + & + \\
\hline \multirow{7}{*}{$\begin{array}{c}\text { Characteristics } \\
\text { on physical examination }\end{array}$} & Pain & - & + & - & + \\
\hline & Swelling & + & + & + & + \\
\hline & Lymphadenopathy & + & + & + & + \\
\hline & Trismus & - & - & - & - \\
\hline & Poor oral hygiene + halitosis & + & + & + & + \\
\hline & Fistula & - & - & + & - \\
\hline & Mobility of the receiver & + & + & + & + \\
\hline \multirow{3}{*}{ Radiographic examination } & Rétro-alvéolar & - & - & - & - \\
\hline & Orthopantomography & + & + & + & + \\
\hline & Computed tomography & - & - & - & - \\
\hline \multirow[t]{3}{*}{ Biologic examination } & $\mathrm{CBC}+$ hemostasis assessment & Abnormal & Abnormal & Abnormal & Abnormal \\
\hline & Sequestrectomy & + & + & + & + \\
\hline & Antibiotic & + & + & + & + \\
\hline \multirow[t]{3}{*}{ Treatment } & Antalgic & - & + & + & + \\
\hline & Antiseptic & + & + & + & + \\
\hline & Antiseptic & + & + & + & + \\
\hline
\end{tabular}

\section{Discussions}

The 4 patients in this series live in a rural area and have no particular history. They were not malnourished, and the biological examinations carried out revealed microcytic hypochromic anemia associated with leuko-lymphocytosis in three out of four patients, negative retroviral serology in all of them, and normal blood glucose, prothrombin, and Activated Partial Thromboplastin (APT) levels, with the exception of the third patient, who presented with a blood glucose level of $1.30 \mathrm{~g} / \mathrm{L}$. However, the etiology of maxillary osteitis reported in the literature showed that these conditions were favored by extreme ages, immunodeficiency, malnutrition and osteopathies [2]. Not all of these etiologies were found in the clinical cases studied. On the other hand, the work of Grott and al on maxillary osteitis noted a possible hereditary etiology after observing the development of the pathology in monozygotic twins, in which no predisposing factor could be identified after clinical, hematological, immunological and microbiological ex- 
amination [3]. The cause remained undetermined and he suggested that hereditary factors might play a role in the pathogenesis of osteitis. The two clinical cases from the same sibling could explain this possible hereditary etiology, but the other children from the same sibling, including the twin of the first case, did not show osteitis.

The notion of odontalgia is always found and the patients took on average 6 months to 24 years before the first consultation, despite the pain and the evolution of the pathology. The problem of access to primary health care for rural populations is always present in Côte d'Ivoire and in Africa; this explains the evolution of osteitis over long periods of time, with an average consultation time ranging from 6 months to over 20 years in this series. The dental infection and the use of traditional procedures by applying decoctions on the swellings and mouthwashes of solution or infusion of various leaf extracts seemed to be a favouring factor. The absence of chewing on the lesioned side, the accumulation of tartar blocks with dental plaque and poor hygiene, were favouring cofactors. During the clinical examination, we noted the presence of swellings of variable size not commensurate with the extent of the sequestration, cutaneous and mucosal fistulas healed or not in the adult and a permanent presence of adenopathies. These endobuccal or cutaneous fistulas in adults were almost non-existent in children in whom ulcerations of the surrounding mucosa were noted. In addition, the amplitude of the mouth opening was normal with a strong halitosis. Mandibular localizations were the most frequent as explained by Diombana who noted that osteitis would come from the increased susceptibility of the maxilla and especially the mandible to osteonecrosis [4].

Radiographic examination often involved panoramic radiography, which showed bone rarefaction and a wet sugar image of the alveolar bone sufficient to establish the diagnosis.

Management begins with the preparation of the oral cavity, preceded by the prescription of antibiotic therapy, usually empirical, based on amoxicillin, with or without metronidazole or fluroquinolone. However, since osteitis is a highly polymicrobial infection, it is composed, according to Ouédraogo, of staphylococci (75\%) and streptococci, pyocyanins, and bacteria (8.3\%). It was therefore indicated that a bone sample be taken for bacteriological analysis before initiating antibiotic therapy because some of the species isolated had the potential to be resistant to certain antibiotics [5].

In the initial stage of osteitis, it would seem to Farah and al, that systemic corticosteroid therapy (prednisone $1 \mathrm{mg} / \mathrm{kg} \times 3 \mathrm{~d}$ ) can stop the evolution towards bone necrosis and the formation of a sequestrum [6]. At a more advanced stage, the treatment of osteitis could be essentially symptomatic and conservative as advocated by Lobato et al. [7]. In the present study, the patients received were from rural areas and consulted late in the stage of bone sequestration. This evolution seemed to be more rapid in children with the risk of loss of the germs of permanent teeth in the sequestration. The size of the sequestration was impres- 
sive, even taking almost all the alveolar bone and mandibular teeth in the adult patient in this series, in whom several very mobile mandibular teeth were extracted and part of the sequester was percutaneously removed by the patient.

After complete removal of the sequestrum, a reorganized mucosa was noted below it. Two sutures of the open alveolar mucosa allowed the surgical wound to be closed and the postoperative prescription was classic based on amoxicillin, paracetamol and antiseptic mouthwash in accordance with Diombala's and Hamed recommendations, which specify that the treatment of osteitis consisted of antiseptic mouthwashes (aqueous chlorhexidine $0,1 \%$ ), antibiotic therapy in case of local superinfection, abscess or fistulization and surgical debridement of the necrotic bone by removal of the splinters or mobile bone sequesters [4] [8]. In some cases of extensive osteonecrosis, only aggressive treatment (interrupted resection of the mandible) was able to achieve resolution of pain and acute infection making prosthetic rehabilitation difficult due to significant bone and tooth loss [9].

The differential diagnosis of odontogenic maxillary osteitis can be made based on osteocondensing or osteolytic tumor diseases or on the presence of certain mutilating oral infectious diseases.

Osteocondensing tumor diseases:

- Fibrous dysplasia characterized by dermatological and neurological lesions.

- Cementoma revealed by X-ray images showing radiculo dental osteocondensation.

Osteolytic tumor diseases:

- Ameloblastoma characterized by its honeycomb-like appear.

- Osteoradionecrosis of the maxilla with signs of radiation therapy.

Mutilating oral infectious diseases like noma, which mainly affects children suffering from malnutrition, combined with poor oral hygiene [8].

Bisphosphonate related osteonecrosis of the jaw as explain respectively. Ruggiero and Sarin in an Updates on bisphosphonates and potential pathobiology of bisphosphonate-induced jaw osteonecrosis [10] [11].

\section{Conclusion}

Osteitis is still frequently diagnosed in our consultations. The epidemiological profile of the subject presenting this pathology today allows putting in common the rural environment, the dental infection, the late consultation, the traditional practices and the bad oral hygiene. Sequesters found are large and involve a significant part of the alveolar bone and permanent or temporary teeth. Spontaneous evolution towards fistulization in adults and the reduction of painful signs, but also the ignorance, the socioeconomic level and the difficulties of access to primary health care in rural populations explain the evolution of these pathologies and the absence of early consultation. Surgical management is simple and can be summarized as the simple harvest of the osteitis but also more complex with an interrupting resection of the mandible making the prosthetic rehabilitation difficult because of the important bone and dental losses. 


\section{Clinical Case No. 1}

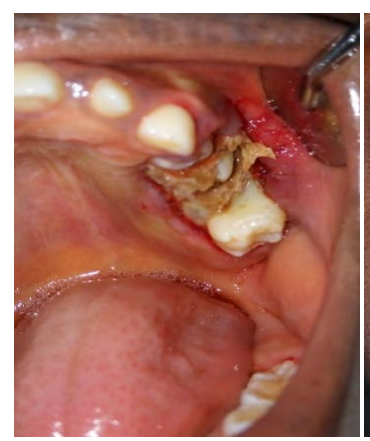

Clinical view before surgery

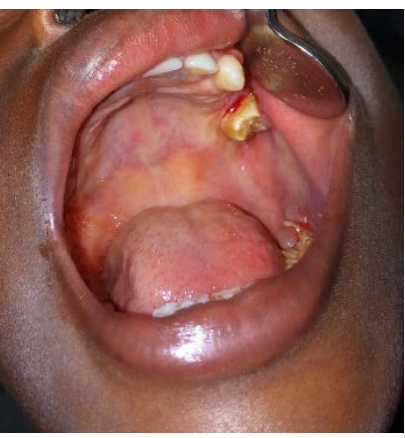

After sequestrectomy

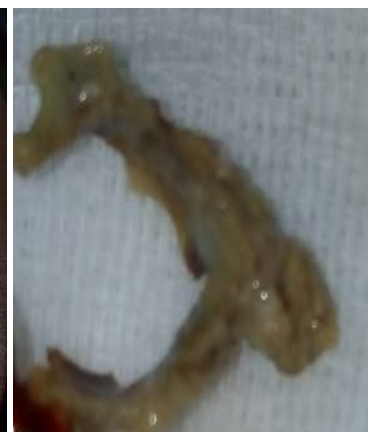

Sequestration

\section{Clinical Case No. 2}

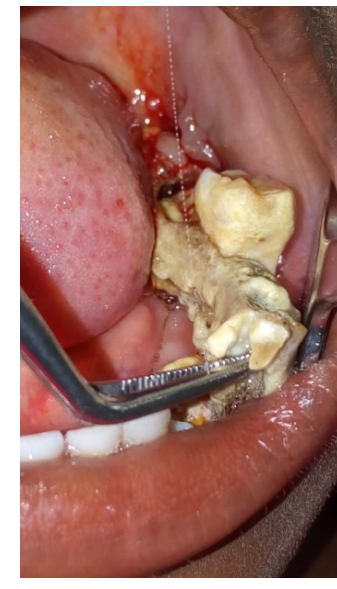

Clinical view
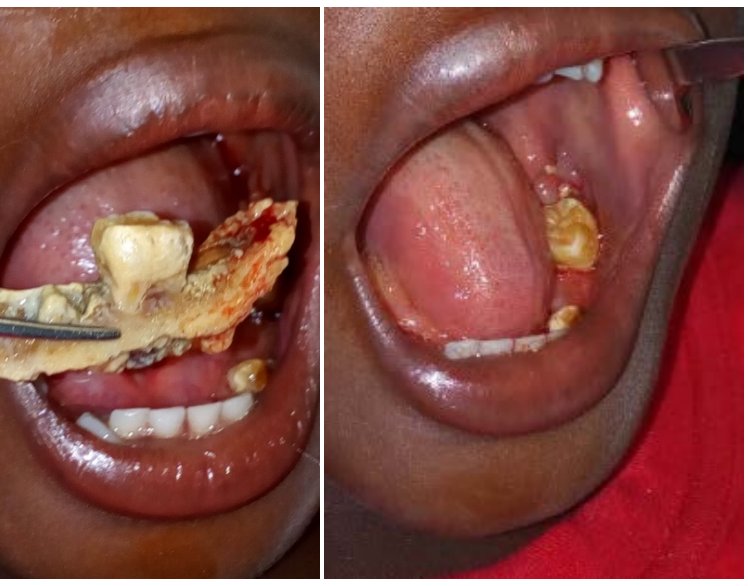

After sequestrectomy

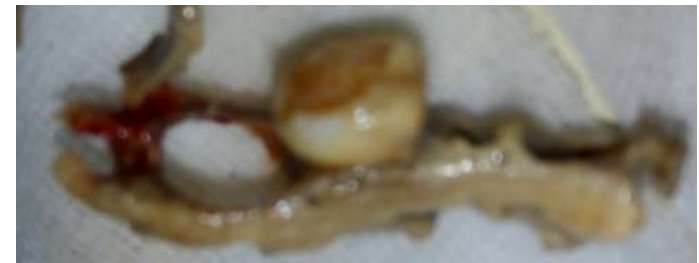

Bone sequestration with 36

Clinical Case No. 3

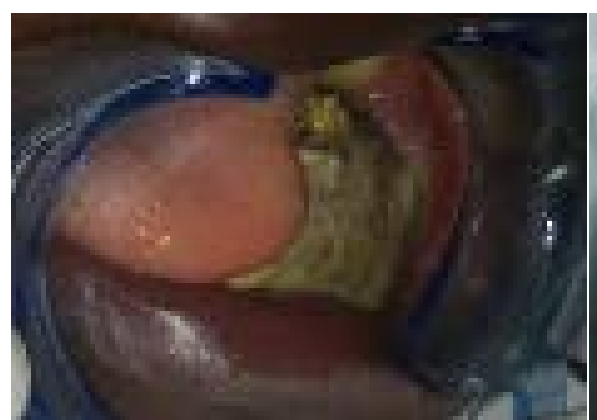

Clinical oral view

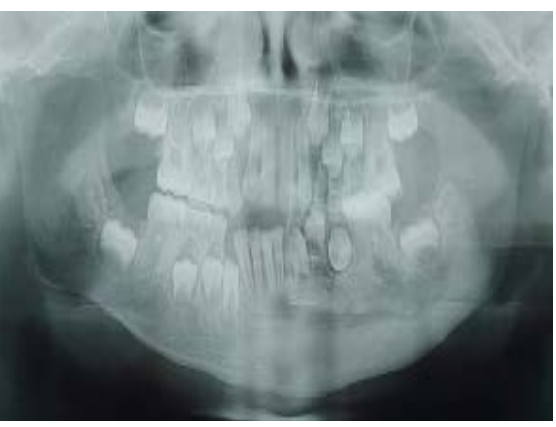

Orthopantomography 


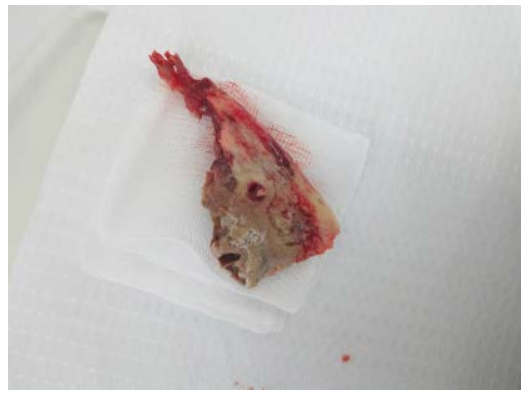

Bone sequestration

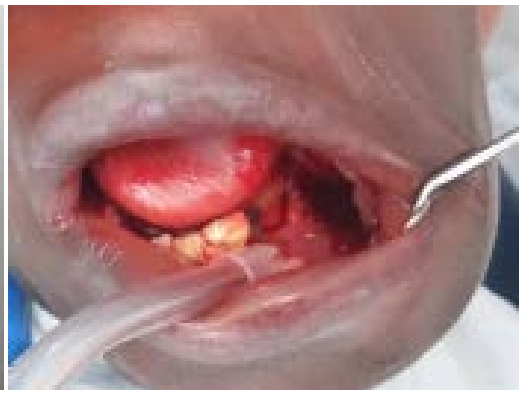

Clinical view after sequestrectomy

\section{Clinical Case No. 4}

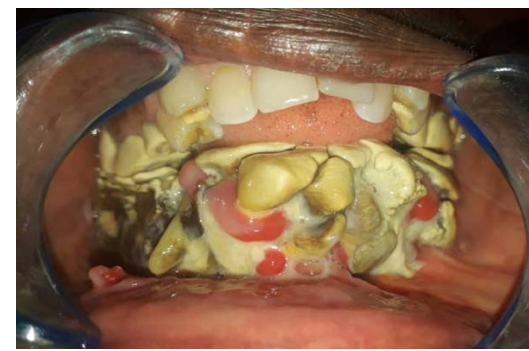

Clinical oral view

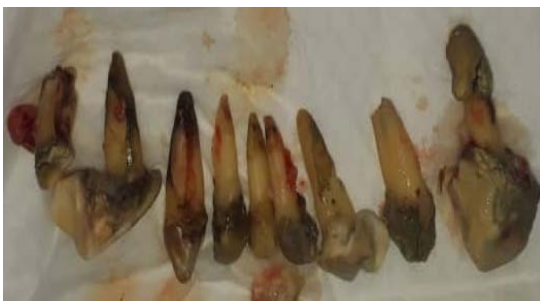

Extracted teeth

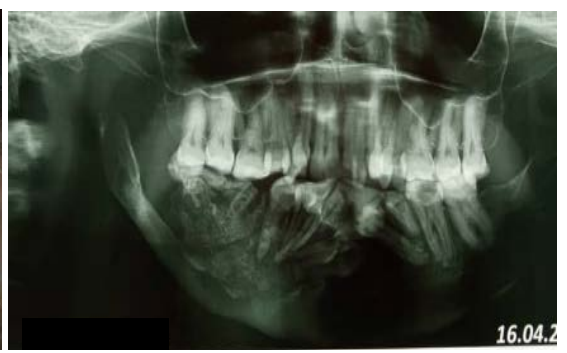

Orthopantomography

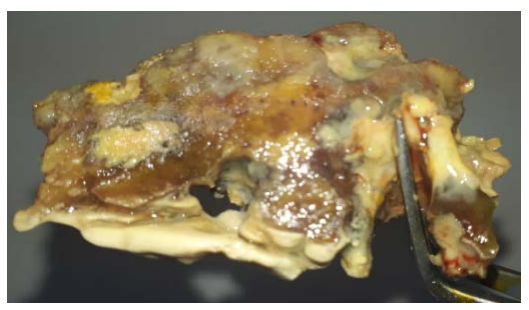

Voluminous bone sequesters

\section{Conflicts of Interest}

The authors declare no conflicts of interest regarding the publication of this paper.

\section{References}

[1] Edouma, B. and Angoh, Y. (2006) Contribution to the Study of Maxillofacial Osteitis in 63 Cases of Osteitis in the Department of Stomatology and Maxillofacial Surgery in Cocody Chu from March 1999 to June 2005. Ph.D. Thesis, University of Cocody, Abidjan.

[2] Maes, J.M., Raoul, G., Omezzine, M. and Ferri, J. (2005) Osteitis of Facial Bones. Enclyclopédie Médico Chirurgicale-Stomatologie, 1, 208-230.

https://doi.org/10.1016/j.emcsto.2005.07.004

[3] Grott, R.H., Van Merkesteyn, J.P.R. and Van Soest, J.J. (1992) Diffuse Sclerosing Osteomyelitis (Chronic Tendoperiostitis) of the Mandible: An 11-Year Follow-Up Report. Oral Surgery, Oral Medicine, Oral Pathology, 74, 557-560. https://doi.org/10.1016/0030-4220(92)90342-N

[4] Diombana, M.L., Mohamed, A.G.A., Kussner, H., Tour, A. and Penneau, M. (1996) Osteitis of the Jaws in the Stomatology Department of the National Hospital of Kati 
(Republic of Mali). About 33 Cases Médecine d'Afrique Noire, 43, 171-173.

[5] Ouédraogo, A., et al. (1999) Osteitis of the Jaws: Our Experience about 25 Observations at the Ouagadougou University Hospital. Medicine d'Afrique Noire, 46, 107-110.

[6] Farah, C.S. and Savage, N.W. (2003) Oral Ulceration with Bone Sequestration. Australian Dental Journal, 48, 61-64. https://doi.org/10.1111/j.1834-7819.2003.tb00011.x

[7] Lobato, J., Mauricio, A.C., Rodrigues, J.M., Cavaleiro, M.V. and Cortez, P.P. (2008) Jaw Avascular Osteonecrosis after Treatment of Multiple Myeloma with Zoledronate. The Journal of Plastic, Reconstructive \& Aesthetic Surgery, 61, 99-106. https://doi.org/10.1016/j.bjps.2006.06.016

[8] Agouzzal, A.A. (2020) Maxillo-Mandibular Osteitis: Retrospective Study and Literature Review. Ph.D. Thesis of Medicine, Université Cadi Ayyad, Marrakech, Maroc.

[9] Reynaud, J., Lacour, M., Grappin, G. and Diop, L. (1966) Diffuse Mandibular Osteitis in Dakar. Bulletin de la Societe de Médecine Afrique Noire de langue française, 11, 20-24.

[10] Ruggiero, S.L., Fantasia, J. and Carlson, E. (2006) Bisphosphonate Related Osteonecrosis of the Jaw: Background and Guidelines for Diagnosis, Staging and Management. Oral Surgery Oral Medicine Oral Pathology Oral Radiology, 102, 433-441. https://doi.org/10.1016/j.tripleo.2006.06.004

[11] Sarin, J., De Rossi, S.S. and Akintoye, S.O. (2008) Updates on Bisphosphonates and Potential Pathobiology of Bisphosphonate-Induced Jaw Osteonecrosis. Oral Diseases, 14, 277-285. https://doi.org/10.1111/j.1601-0825.2007.01381.x 